\title{
Bifurcation analysis of a two-species competitive discrete model of plankton allelopathy
}

Daiyong $\mathrm{Wu}^{1^{*}}$ and Hai Zhang ${ }^{1,2}$

"Correspondence:

wudy9901@163.com

1 Department of Mathematics,

Anqing Normal University, Anqing,

Anhui 246133, China

Full list of author information is

available at the end of the article

\begin{abstract}
This paper studies the dynamical behaviors of a two-species competitive discrete model of plankton allelopathy. The system undergoes a flip bifurcation as we see by using the center manifold theorem and bifurcation theory. Numerical simulations not only illustrate our results, but they also exhibit the complex dynamical behaviors of the system, such as the period-doubling bifurcation in periods $2,4,8$, and 16 , and chaotic sets.
\end{abstract}

Keywords: plankton allelopathy; competitive system; discrete; flip bifurcation

\section{Introduction}

The study of tremendous fluctuations in the abundance of many phytoplankton communities is an important subject in aquatic ecology. These changes of size and density of phytoplankton have been attributed to several factors, such as physical factors, variation of necessary nutrients, or a combination of these by various workers (see $c f$. [1-5]). Another important observation made by many workers is that the increased population of one species might affect the growth of another species or several other species by the production of allelopathic toxins or stimulators, thus influencing seasonal succession [6].

The traditional Lotka-Volterra two-species competitive system can be expressed as follows:

$$
\left\{\begin{array}{l}
\dot{x}_{1}(t)=x_{1}(t)\left(K_{1}-\alpha_{1} x_{1}(t)-\beta_{12} x_{2}(t)\right), \\
\dot{x}_{2}(t)=x_{2}(t)\left(K_{2}-\alpha_{2} x_{2}(t)-\beta_{21} x_{1}(t)\right),
\end{array}\right.
$$

where $x_{1}(t), x_{2}(t)$ are the population densities (number of cells per liter) of two competing species; $K_{1}, K_{2}$ are the rates of cell proliferation per hour; $\alpha_{1}, \alpha_{2}$ are the rate of intra-specific competition of first and second species, respectively; $\beta_{12}, \beta_{21}$ are the rate of inter specific competition of first and second species respectively and $\frac{K_{1}}{\alpha_{1}}, \frac{K_{2}}{\alpha_{2}}$ are environmental carrying capacities (representing number of cells per liter). The units of $\alpha_{1}, \alpha_{2}, \beta_{12}$ and $\beta_{21}$ are per hour per cell and the unit of time is hours.

Maynard [7] and Chattopadhyay [8] modified the system (1.1) by considering that each species produced a substance toxic to the other, but only when the other is present. Then

○2014 Wu and Zhang; licensee Springer. This is an Open Access article distributed under the terms of the Creative Commons Attribution License (http://creativecommons.org/licenses/by/2.0), which permits unrestricted use, distribution, and reproduction in any medium, provided the original work is properly cited. 
the system (1.1) can be written as

$$
\left\{\begin{array}{l}
\dot{x}_{1}(t)=x_{1}(t)\left(K_{1}-\alpha_{1} x_{1}(t)-\beta_{12} x_{2}(t)-\gamma_{1} x_{1}(t) x_{2}(t)\right) \\
\dot{x}_{2}(t)=x_{2}(t)\left(K_{2}-\alpha_{2} x_{2}(t)-\beta_{21} x_{1}(t)-\gamma_{2} x_{1}(t) x_{2}(t)\right)
\end{array}\right.
$$

where $\gamma_{1}$ and $\gamma_{2}$ are the rates of toxic inhibition of the first species by the second and vice versa, respectively, and $K_{1}, K_{2}, \alpha_{1}, \alpha_{2}, \beta_{12}, \beta_{21}, \gamma_{1}$, and $\gamma_{2}$ are positive constants.

On the other hands, many scholars have paid attention to the discrete population models, since the discrete-time models governed by discrete systems are more appropriate than the continuous ones when the populations have nonoverlapping generations ( $c f$. [912]). Moreover, since the discrete-time models can also provide efficient computational models of continuous models for numerical simulations, it is reasonable to study discretetime models governed by discrete systems.

In this paper, we apply the forward Euler scheme to the system (1.2) and obtain the twospecies competitive discrete-time system of plankton allelopathy as follows:

$$
\left(\begin{array}{l}
x_{1} \\
x_{2}
\end{array}\right) \rightarrow\left(\begin{array}{c}
x_{1}+\delta x_{1}\left[K_{1}-\alpha_{1} x_{1}-\beta_{12} x_{2}-\gamma_{1} x_{1} x_{2}\right] \\
x_{2}+\delta x_{2}\left[K_{2}-\alpha_{2} x_{2}-\beta_{21} x_{1}-\gamma_{2} x_{1} x_{2}\right]
\end{array}\right),
$$

where $\delta>0$ is the step size.

The dynamical behaviors of discrete system of plankton allelopathy have been investigated in the mathematics literature ( $c f$. [13-15]). The purpose of this paper is to investigate the bifurcation and chaos of the map $(1.3)$ by using bifurcation theory $(c f .[16,17])$ and center manifold theory ( $c f .[16-18])$. Meanwhile, numerical simulations are presented not only to illustrate our results with the theoretical analysis, but also to exhibit the complex dynamical behaviors.

This paper is organized as follows. In Section 2, we discuss the existence and stability of the positive fixed points for the system (1.3). In Section 3, we show that there exist some values of parameters such that the system (1.3) undergoes the flip bifurcation. In Section 4, we present numerical simulations which illustrate our results with the theoretical analysis. A brief discussion is given in Section 5.

\section{Fixed points and stability analysis}

Recently, Samanta [19] further investigated the system (1.2) and showed that a unique interior equilibrium point exists if one of 22 conditions holds. Similarly, the system (1.3) has a unique positive fixed point if one of the 22 conditions holds. In this paper, we only restrict our attention to the following condition:

$$
\frac{\alpha_{1}}{\beta_{21}}>\frac{K_{1}}{K_{2}}>\frac{\beta_{12}}{\alpha_{2}}, \quad \frac{\alpha_{1}}{\beta_{21}}>\frac{\gamma_{1}}{\gamma_{2}}>\frac{\beta_{12}}{\alpha_{2}} .
$$

Throughout this paper, we always assume that the condition (2.1) holds. Then the system (1.3) has the unique positive fixed point $E\left(x_{1}^{*}, x_{2}^{*}\right)$, where

$$
\begin{aligned}
& x_{1}^{*}=\frac{C_{1}+\sqrt{C_{1}^{2}+4\left(\gamma_{2} \alpha_{1}-\gamma_{1} \beta_{21}\right)\left(K_{1} \alpha_{2}-K_{2} \beta_{12}\right)}}{2\left(\gamma_{2} \alpha_{1}-\gamma_{1} \beta_{21}\right)}, \\
& x_{2}^{*}=\frac{C_{2}+\sqrt{C_{2}^{2}+4\left(\gamma_{1} \alpha_{2}-\gamma_{2} \beta_{12}\right)\left(K_{2} \alpha_{1}-K_{1} \beta_{21}\right)}}{2\left(\gamma_{1} \alpha_{2}-\gamma_{2} \beta_{12}\right)},
\end{aligned}
$$


and

$$
\begin{aligned}
& C_{1}=K_{1} \gamma_{2}-K_{2} \gamma_{1}+\beta_{12} \beta_{21}-\alpha_{1} \alpha_{2}, \\
& C_{2}=K_{2} \gamma_{1}-K_{1} \gamma_{2}+\beta_{12} \beta_{21}-\alpha_{1} \alpha_{2}
\end{aligned}
$$

Now we study the stability of the fixed point $E\left(x_{1}^{*}, x_{2}^{*}\right)$. Note that the local stability of a fixed point $\left(x_{1}, x_{2}\right)$ is determined by the modules of the eigenvalues of the characteristic equation at the fixed point. The generalized Jacobian matrix $J\left(x_{1}, x_{2}\right)$ of the system (1.3) evaluated at any point $\left(x_{1}, x_{2}\right)$ is given by

$$
J\left(x_{1}, x_{2}\right)=\left(\begin{array}{ll}
g_{11} & g_{12} \\
g_{21} & g_{22}
\end{array}\right),
$$

where

$$
\begin{aligned}
& g_{11}=1+\delta\left(K_{1}-2 \alpha_{1} x_{1}-\beta_{12} x_{2}-2 \gamma_{1} x_{1} x_{2}\right), \\
& g_{12}=\delta x_{1}\left(-\beta_{12}-\gamma_{1} x_{1}\right), \\
& g_{21}=\delta x_{2}\left(-\beta_{21}-\gamma_{2} x_{2}\right), \\
& g_{22}=1+\delta\left(K_{2}-2 \alpha_{2} x_{2}-\beta_{21} x_{1}-2 \gamma_{2} x_{1} x_{2}\right) .
\end{aligned}
$$

The characteristic equation of Jacobian matrix can be written as

$$
\lambda^{2}-\operatorname{Tr}\left(J\left(x_{1}, x_{2}\right)\right) \lambda+\operatorname{Det}\left(J\left(x_{1}, x_{2}\right)\right)=0
$$

where

$$
\begin{aligned}
\operatorname{Tr}\left(J\left(x_{1}, x_{2}\right)\right)=2 & +\delta\left[\left(K_{1}-2 \alpha_{1} x_{1}-\beta_{12} x_{2}-2 \gamma_{1} x_{1} x_{2}\right)\right. \\
+ & \left.\left(K_{2}-2 \alpha_{2} x_{2}-\beta_{21} x_{1}-2 \gamma_{2} x_{1} x_{2}\right)\right], \\
\operatorname{Det}\left(J\left(x_{1}, x_{2}\right)\right)= & \left(1+\delta\left(K_{1}-2 \alpha_{1} x_{1}-\beta_{12} x_{2}-2 \gamma_{1} x_{1} x_{2}\right)\right) \\
& \times\left(1+\delta\left(K_{2}-2 \alpha_{2} x_{2}-\beta_{21} x_{1}-2 \gamma_{2} x_{1} x_{2}\right)\right) \\
& -\delta^{2} x_{1} x_{2}\left(\beta_{12}+\gamma_{1} x_{1}\right)\left(\beta_{21}+\gamma_{2} x_{2}\right) .
\end{aligned}
$$

In order to study the stability of the fixed points $E\left(x_{1}^{*}, x_{2}^{*}\right)$ of the system (1.3), we first give the following lemma, which can easily be proved by the relations between roots and coefficients of a quadratic equation.

Lemma 2.1 Let $F(\lambda)=\lambda^{2}+P \lambda+Q$, where $P$ and $Q$ are constants. Suppose that $F(1)>0$, $\lambda_{1}, \lambda_{2}$ are two roots of $F(\lambda)=0$. Then

(i) $\left|\lambda_{1}\right|<1$ and $\left|\lambda_{2}\right|<1$ if and only if $F(-1)>0$ and $Q<1$;

(ii) $\left|\lambda_{1}\right|<1$ and $\left|\lambda_{2}\right|>1$ (or $\left|\lambda_{1}\right|>1$ and $\left|\lambda_{2}\right|<1$ ) if and only if $F(-1)<0$;

(iii) $\left|\lambda_{1}\right|>1$ and $\left|\lambda_{2}\right|>1$ if and only if $F(-1)>0$ and $Q>1$;

(iv) $\lambda_{1}=-1$ and $\left|\lambda_{2}\right| \neq 1$ if and only if $F(-1)=0$ and $Q \neq 1$;

(v) $\lambda_{1}$ and $\lambda_{2}$ are complex and $\left|\lambda_{1}\right|=1$ and $\left|\lambda_{2}\right|=1$ if and only if $P^{2}-4 Q<0$ and $Q=1$. 
Let $\lambda_{1}$ and $\lambda_{2}$ be two roots of (2.2), which are called eigenvalues of the fixed point $\left(x_{1}, x_{2}\right)$. We recall some definitions of topological types for a fixed point $\left(x_{1}, x_{2}\right) .\left(x_{1}, x_{2}\right)$ is called a sink if $\left|\lambda_{1}\right|<1$ and $\left|\lambda_{2}\right|<1$. A sink is locally asymptotic stable. $\left(x_{1}, x_{2}\right)$ is called a source if $\left|\lambda_{1}\right|>1$ and $\left|\lambda_{2}\right|>1$. A source is locally unstable. $\left(x_{1}, x_{2}\right)$ is called a saddle if $\left|\lambda_{1}\right|>1$ and $\left|\lambda_{2}\right|<1$ (or $\left|\lambda_{1}\right|<1$ and $\left.\left|\lambda_{2}\right|>1\right)$. $\left(x_{1}, x_{2}\right)$ is called non-hyperbolic if either $\left|\lambda_{1}\right|=1$ or $\left|\lambda_{2}\right|=1$.

Now, we discuss the stability of fixed point $E\left(x_{1}^{*}, x_{2}^{*}\right)$. The characteristic equation of the fixed pointed $E\left(x_{1}^{*}, x_{2}^{*}\right)$ can be represented as

$$
F(\lambda)=\lambda^{2}-\operatorname{Tr}\left(J\left(x_{1}^{*}, x_{2}^{*}\right)\right) \lambda+\operatorname{Det}\left(J\left(x_{1}^{*}, x_{2}^{*}\right)\right)=0,
$$

where $\operatorname{Tr}\left(J\left(x_{1}^{*}, x_{2}^{*}\right)\right)=2-\delta A$ and $\operatorname{Det}\left(J\left(x_{1}^{*}, x_{2}^{*}\right)\right)=1+\delta^{2} B-\delta A$,

$$
\begin{aligned}
& A=\alpha_{1} x_{1}^{*}+\gamma_{1} x_{1}^{*} x_{2}^{*}+\alpha_{2} x_{2}^{*}+\gamma_{2} x_{1}^{*} x_{2}^{*}, \\
& B=x_{1}^{*} x_{2}^{*}\left(\alpha_{1} \alpha_{2}+\alpha_{2} \gamma_{1} x_{2}^{*}+\alpha_{1} \gamma_{2} x_{1}^{*}-\beta_{12} \beta_{21}-\beta_{12} \gamma_{2} x_{2}^{*}-\beta_{21} \gamma_{1} x_{1}^{*}\right) .
\end{aligned}
$$

By the condition (2.1), we can obtain $B>0$. Moreover,

$$
A^{2}-4 B=\left[\alpha_{1} x_{1}^{*}-\alpha_{2} x_{2}^{*}+\left(\gamma_{1}-\gamma_{2}\right) x_{1}^{*} x_{2}^{*}\right]^{2}+4\left(\beta_{12} \beta_{21}+\beta_{12} \gamma_{2} x_{2}^{*}+\beta_{21} \gamma_{1} x_{1}^{*}+\gamma_{1} \gamma_{2} x_{1}^{*} x_{2}^{*}\right)>0 .
$$

Clearly,

$$
F(1)=1-(2-\delta A)+1+\delta^{2} B-\delta A=\delta^{2} B>0
$$

and

$$
F(-1)=4-2 \delta A+\delta^{2} B
$$

Note that $\triangle_{1}=A^{2}-4 B>0$, so there exist $\delta_{1}=\frac{A-\sqrt{\Delta_{1}}}{B}$ and $\delta_{2}=\frac{A+\sqrt{\Delta_{1}}}{B}$ leading to $F(-1)=0$.

Regarding the stability of $E\left(x_{1}^{*}, x_{2}^{*}\right)$, we have the following results.

Since $A^{2}-4 B>0, F(\lambda)=0$ has two unequal real roots $\lambda_{1}$ and $\lambda_{2}$. Furthermore, we obtain the following.

$\left(\mathrm{A}_{1}\right)$ If $0<\delta<\delta_{1}$, then $F(-1)>0$ and $\operatorname{Det}\left(J\left(x_{1}^{*}, x_{2}^{*}\right)\right)=1+\delta^{2} B-\delta A<1$. By Lemma 2.1 we have $\left|\lambda_{1}\right|<1$ and $\left|\lambda_{2}\right|<1$. Therefore, $E\left(x_{1}^{*}, x_{2}^{*}\right)$ is a sink.

$\left(\mathrm{A}_{2}\right)$ If $\delta>\delta_{2}$, then $F(-1)>0$ and $\operatorname{Det}\left(J\left(x_{1}^{*}, x_{2}^{*}\right)\right)=1+\delta^{2} B-\delta A>1$. By Lemma 2.1 we have $\left|\lambda_{1}\right|>1$ and $\left|\lambda_{2}\right|>1$. Therefore, $E\left(x_{1}^{*}, x_{2}^{*}\right)$ is a source.

$\left(\mathrm{A}_{3}\right)$ If $\delta=\delta_{1}$ or $\delta_{2}$, then $F(-1)=0$ and $\operatorname{Det}\left(J\left(x_{1}^{*}, x_{2}^{*}\right)\right)=1+\delta^{2} B-\delta A \neq 1$. By Lemma 2.1 we have $\lambda_{1}=-1$ and $\left|\lambda_{2}\right| \neq 1$. Therefore, $E\left(x_{1}^{*}, x_{2}^{*}\right)$ is non-hyperbolic.

$\left(\mathrm{A}_{4}\right)$ If $\delta_{1}<\delta<\delta_{2}$, then $F(-1)<0$. By Lemma 2.1 we have $\left|\lambda_{1}\right|<1$ and $\left|\lambda_{2}\right|>1$ (or $\left|\lambda_{1}\right|>1$ and $\left.\left|\lambda_{2}\right|<1\right)$. Therefore, $E\left(x_{1}^{*}, x_{2}^{*}\right)$ is a saddle.

From the above analysis, we obtain the result that for the fixed point $E\left(x_{1}^{*}, x_{2}^{*}\right)$, if $\left(K_{1}, K_{2}, \alpha_{1}, \alpha_{2}, \beta_{12}, \beta_{21}, \gamma_{1}, \gamma_{2}, \delta\right) \in M_{i}, i=1,2$, where $M_{i}=\left\{\left(K_{j}, \alpha_{j}, \beta_{12}, \beta_{21}, \gamma_{j}, \delta\right): A^{2}-4 B>\right.$ $\left.0, \delta=\delta_{i}, K_{j}>0, \alpha_{j}>0, \gamma_{j}>0, \beta_{12}>0, \beta_{21}>0, j=1,2\right\}$, then one of the two eigenvalues of the positive fixed point $E\left(x_{1}^{*}, x_{2}^{*}\right)$ is -1 and the other is neither 1 nor -1 . Therefore, there may be a flip bifurcation of $E\left(x_{1}^{*}, x_{2}^{*}\right)$ if the parameters vary in the small neighborhood of $M_{i}$, $i=1,2$. 
Remark Since $F(1)>0$, one can see that 1 is not the eigenvalue of the positive fixed point $E\left(x_{1}^{*}, x_{2}^{*}\right)$. Therefore, fold bifurcations, transcritical bifurcations, and pitchfork bifurcations do not occur at the positive fixed point $E\left(x_{1}^{*}, x_{2}^{*}\right)$. Similarly, since $A^{2}-4 B>0, F(\lambda)=0$, and there does not exist a pair of conjugate complex roots with modulus 1 . Hence, the Neimark-Sacker bifurcation does not occur at the positive fixed point $E\left(x_{1}^{*}, x_{2}^{*}\right)$.

\section{Flip bifurcation}

In this section, we choose parameter $\delta$ as a bifurcation parameter to study the flip bifurcation of $E\left(x_{1}^{*}, x_{2}^{*}\right)$ by using the center manifold theorem and the bifurcation theory in [1618]. For convenience, for a function $f\left(x_{1}, x_{2}, \ldots, x_{n}\right)$, we denote by $f_{x_{i}}, f_{x_{i} x_{j}}$, and $f_{x_{i} x_{j} x_{k}}$ the first order, second order and the third order partial derivative of $f\left(x_{1}, x_{2}, \ldots, x_{n}\right)$, respectively.

We first discuss the flip bifurcation of the system (1.3) at $E\left(x_{1}^{*}, x_{2}^{*}\right)$ when the parameter varies in a small neighborhood of $M_{1}$. Similar arguments can be applied to the other case, $M_{2}$. Taking the parameters $\left(K_{1}, K_{2}, \alpha_{1}, \alpha_{2}, \beta_{12}, \beta_{21}, \gamma_{1}, \gamma_{2}, \delta\right) \in M_{1}$ arbitrarily, we consider the system (1.3) at $E\left(x_{1}^{*}, x_{2}^{*}\right)$.

From $\left(K_{1}, K_{2}, \alpha_{1}, \alpha_{2}, \beta_{12}, \beta_{21}, \gamma_{1}, \gamma_{2}, \delta\right) \in M_{1}$, we have $\delta=\delta_{1}$. Giving a perturbation $\delta^{*}$ of the parameter $\delta$, we consider a perturbation of the system (1.3) as follows:

$$
\left\{\begin{array}{l}
x_{1} \rightarrow x_{1}+\left(\delta_{1}+\delta^{*}\right) x_{1}\left[K_{1}-\alpha_{1} x_{1}-\beta_{12} x_{2}-\gamma_{1} x_{1} x_{2}\right] \\
x_{2} \rightarrow x_{2}+\left(\delta_{1}+\delta^{*}\right) x_{2}\left[K_{2}-\alpha_{2} x_{2}-\beta_{21} x_{1}-\gamma_{2} x_{1} x_{2}\right]
\end{array}\right.
$$

where $\delta^{*}$ is a small perturbation parameter.

Let $y_{1}=x_{1}-x_{1}^{*}$ and $y_{2}=x_{2}-x_{2}^{*}$, and transform the fixed point $E\left(x_{1}^{*}, x_{2}^{*}\right)$ of the map (3.1) to the origin. Substituting $x_{1}=y_{1}+x_{1}^{*}$ and $x_{2}=y_{2}+x_{2}^{*}$ into the map (3.1), we obtain

$$
\left(\begin{array}{l}
y_{1} \\
y_{2}
\end{array}\right) \rightarrow\left(\begin{array}{c}
a_{11} y_{1}+a_{12} y_{2}+a_{13} y_{1} y_{2}+a_{14} y_{1}^{2}+a_{15} y_{1}^{2} y_{2} \\
+b_{1} \delta^{*} y_{1}+b_{2} \delta^{*} y_{2}+b_{3} \delta^{*} y_{1} y_{2}+b_{4} \delta^{*} y_{1}^{2}+b_{5} \delta^{*} y_{1}^{2} y_{2} \\
a_{21} y_{1}+a_{22} y_{2}+a_{23} y_{1} y_{2}+a_{24} y_{2}^{2}+a_{25} y_{1} y_{2}^{2} \\
+c_{1} \delta^{*} y_{2}+c_{2} \delta^{*} y_{1}+c_{3} \delta^{*} y_{1} y_{2}+c_{4} \delta^{*} y_{2}^{2}+c_{5} \delta^{*} y_{1} y_{2}^{2}
\end{array}\right)
$$

where

$$
\begin{aligned}
& a_{11}=1-\delta_{1} x_{1}^{*}\left(\alpha_{1}+\gamma_{1} x_{2}^{*}\right), \quad a_{12}=-\delta_{1} x_{1}^{*}\left(\beta_{12}+\gamma_{1} x_{1}^{*}\right), \quad a_{13}=-\delta_{1}\left(\beta_{12}+2 \gamma_{1} x_{1}^{*}\right), \\
& a_{14}=-\delta_{1}\left(\alpha_{1}+\gamma_{1} x_{2}^{*}\right), \quad a_{15}=-\gamma_{1} \delta_{1}, \quad b_{1}=-x_{1}^{*}\left(\alpha_{1}+\gamma_{1} x_{2}^{*}\right), \\
& b_{2}=-x_{1}^{*}\left(\beta_{12}+\gamma_{1} x_{1}^{*}\right), \quad b_{3}=-\left(\beta_{12}+2 \gamma_{1} x_{1}^{*}\right), \quad b_{4}=-\left(\alpha_{1}+\gamma_{1} x_{2}^{*}\right), \\
& b_{5}=-\gamma_{1}, \quad a_{21}=-\delta_{1} x_{2}^{*}\left(\beta_{21}+\gamma_{2} x_{2}^{*}\right), \\
& a_{22}=1-\delta_{1} x_{2}^{*}\left(\alpha_{2}+\gamma_{2} x_{1}^{*}\right), \quad a_{23}=-\delta_{1}\left(\beta_{21}+2 \gamma_{2} x_{2}^{*}\right), \quad a_{24}=-\delta_{1}\left(\alpha_{2}+\gamma_{2} x_{1}^{*}\right), \\
& a_{25}=-\gamma_{2} \delta_{1}, \quad c_{1}=-x_{2}^{*}\left(\alpha_{2}+\gamma_{2} x_{1}^{*}\right), \quad c_{2}=-x_{2}^{*}\left(\beta_{21}+\gamma_{2} x_{2}^{*}\right), \\
& c_{3}=-\left(\beta_{21}+2 \gamma_{2} x_{2}^{*}\right), \quad c_{4}=-\left(\alpha_{2}+\gamma_{2} x_{1}^{*}\right), \quad b_{5}=-\gamma_{2} .
\end{aligned}
$$

Let $T_{1}=\left(\begin{array}{ll}a_{11} & a_{12} \\ a_{21} & a_{22}\end{array}\right)$. By calculating, we find that the eigenvalues and the corresponding eigenvectors of matrix $T_{1}$ are

$$
\lambda_{1}=-1, \quad \lambda_{2}=3-\delta_{1} A,
$$


with $\left|\lambda_{1}\right|=1,\left|\lambda_{2}\right| \neq 1$ and $\left(d_{1}, d_{2}\right)^{T}=\left(a_{12},-1-a_{11}\right)^{T},\left(d_{3}, d_{4}\right)^{T}=\left(a_{12}, \lambda_{2}-a_{11}\right)^{T}$, respectively.

Let matrix $T_{2}=\left(\begin{array}{ll}d_{1} & d_{3} \\ d_{2} & d_{4}\end{array}\right)$, then $T_{2}$ is invertible. Using a translation $\left(\begin{array}{l}y_{1} \\ y_{2}\end{array}\right)=T_{2}\left(\begin{array}{l}\widetilde{x}_{1} \\ \widetilde{x}_{2}\end{array}\right)$, the map (3.2) becomes

$$
\left(\begin{array}{l}
\tilde{x}_{1} \\
\tilde{x}_{2}
\end{array}\right) \rightarrow\left(\begin{array}{cc}
-1 & 0 \\
0 & \lambda_{2}
\end{array}\right)\left(\begin{array}{l}
\tilde{x}_{1} \\
\tilde{x}_{2}
\end{array}\right)+\left(\begin{array}{l}
f\left(\widetilde{x}_{1}, \widetilde{x}_{2}, \delta^{*}\right) \\
g\left(\widetilde{x}_{1}, \widetilde{x}_{2}, \delta^{*}\right)
\end{array}\right),
$$

where

$$
\begin{aligned}
f\left(\widetilde{x}_{1}, \widetilde{x}_{2}, \delta^{*}\right)= & \frac{\left(\lambda-a_{11}\right)}{a_{12}(\lambda+1)}\left(a_{13} y_{1} y_{2}+a_{14} y_{1}^{2}+a_{15} y_{1}^{2} y_{2}+b_{1} \delta^{*} y_{1}\right. \\
& \left.+b_{2} \delta^{*} y_{2}+b_{3} \delta^{*} y_{1} y_{2}+b_{4} \delta^{*} y_{1}^{2}+b_{5} \delta^{*} y_{1}^{2} y_{2}\right) \\
& -\frac{1}{\lambda+1}\left(a_{23} y_{1} y_{2}+a_{24} y_{2}^{2}+a_{25} y_{1} y_{2}^{2}+c_{1} \delta^{*} y_{2}\right. \\
& \left.+c_{2} \delta^{*} y_{1}+c_{3} \delta^{*} y_{1} y_{2}+c_{4} \delta^{*} y_{2}^{2}+c_{5} \delta^{*} y_{1} y_{2}^{2}\right), \\
f_{2}\left(\widetilde{x}_{1}, \widetilde{x}_{2}, \delta^{*}\right)= & \frac{\left(1+a_{11}\right)}{a_{12}(\lambda+1)}\left(a_{13} y_{1} y_{2}+a_{14} y_{1}^{2}+a_{15} y_{1}^{2} y_{2}+b_{1} \delta^{*} y_{1}\right. \\
& \left.+b_{2} \delta^{*} y_{2}+b_{3} \delta^{*} y_{1} y_{2}+b_{4} \delta^{*} y_{1}^{2}+b_{5} \delta^{*} y_{1}^{2} y_{2}\right) \\
& +\frac{1}{\lambda+1}\left(a_{23} y_{1} y_{2}+a_{24} y_{2}^{2}+a_{25} y_{1} y_{2}^{2}+c_{1} \delta^{*} y_{2}\right. \\
& \left.+c_{2} \delta^{*} y_{1}+c_{3} \delta^{*} y_{1} y_{2}+c_{4} \delta^{*} y_{2}^{2}+c_{5} \delta^{*} y_{1} y_{2}^{2}\right),
\end{aligned}
$$

and

$$
\begin{aligned}
& y_{1}=a_{12}\left(\widetilde{x}_{1}+\widetilde{x}_{2}\right), \quad y_{2}=-\widetilde{x}_{1}+\lambda_{2} \widetilde{x}_{2}-a_{11}\left(\widetilde{x}_{1}+\widetilde{x}_{2}\right), \quad y_{1}^{2}=a_{12}^{2}\left(\widetilde{x}_{1}^{2}+2 \widetilde{x}_{1} \widetilde{x}_{2}+\widetilde{x}_{2}^{2}\right), \\
& y_{1} y_{2}=-a_{12}\left(1+a_{11}\right) \widetilde{x}_{1}^{2}+\left[a_{12}\left(\lambda_{2}-a_{11}\right)-a_{12}\left(1+a_{11}\right)\right] \widetilde{x}_{1} \widetilde{x}_{2}+a_{12}\left(\lambda_{2}-a_{11}\right) \widetilde{x}_{2}^{2} .
\end{aligned}
$$

Now, we determine the center manifold $W^{c}(0,0,0)$ of $(3.3)$ at the fixed point $(0,0)$ in a small neighborhood of $\delta^{*}=0$. Note that $f$ and $g$ are of class $C^{K+1}$-functions for some $k \geq 1$ and $f(0,0,0)=0, g(0,0,0)=0, f_{\widetilde{x}_{i}}(0,0,0)=0, g_{\widetilde{x}_{i}}(0,0,0)=0, i=1,2$. Hence, based on the center manifold theorem [18], we know there exists a center manifold

$$
W^{c}(0,0,0)=\left\{\left(\widetilde{x}_{1}, \widetilde{x}_{2}, \delta^{*}\right) \in R^{3}: \widetilde{x}_{2}=h\left(\widetilde{x}_{1}, \delta^{*}\right), h(0,0)=0, \operatorname{Dh}(0,0)=0\right\}
$$

for $\widetilde{x}_{1}$ and $\delta^{*}$ sufficiently small. The form of the center manifold can be approximately represented as follows:

$$
h\left(\widetilde{x}_{1}, \delta^{*}\right)=a_{1} \widetilde{x}_{1}^{2}+a_{2} \widetilde{x}_{1} \delta^{*}+a_{3} \delta^{* 2}+o\left(\left(\left|\widetilde{x}_{1}\right|+\left|\delta^{*}\right|\right)^{2}\right),
$$

where $o\left(\left(\left|\widetilde{x}_{1}\right|+\left|\delta^{*}\right|\right)^{2}\right)$ is a function with order at least three in their variables $\left(\widetilde{x}_{1}, \delta^{*}\right)$. Moreover, the center manifold must satisfy

$$
h\left(-\widetilde{x}_{1}+f\left(\widetilde{x}_{1}, h\left(\widetilde{x}_{1}, \delta^{*}\right), \delta^{*}\right), \delta^{*}\right)-\lambda \lambda_{2} h\left(\widetilde{x}_{1}, \delta^{*}\right)-g\left(\widetilde{x}_{1}, h\left(\widetilde{x}_{1}, \delta^{*}\right), \delta^{*}\right)=0 .
$$


By equating coefficients of like powers to zero, we obtain

$$
\begin{aligned}
& a_{1}=\frac{1}{1-\lambda_{2}^{2}}\left(a_{11}+1\right)\left[a_{12} a_{14}-a_{13}\left(1+a_{11}\right)-a_{12} a_{23}\right], \\
& a_{2}=\frac{-b_{1}\left(a_{11}+1\right)-c_{2} a_{12}+c_{1}\left(a_{11}+1\right)}{\left(\lambda_{2}+1\right)^{2}}+\frac{b_{2}\left(a_{11}+1\right)^{2}}{a_{12}\left(\lambda_{2}+1\right)^{2}}, \quad a_{3}=0 .
\end{aligned}
$$

Therefore, we consider the map which is the map (3.3) restricted to the center manifold $W^{c}(0,0,0)$ :

$$
G: \tilde{x}_{1} \rightarrow-\widetilde{x}_{1}+h_{1} \tilde{x}_{1}^{2}+h_{2} \tilde{x}_{1} \delta^{*}+h_{3} \tilde{x}_{1}^{2} \delta^{*}+h_{4} \tilde{x}_{1} \delta^{* 2}+h_{5} \tilde{x}_{1}^{3}+o\left(\left(\left|\tilde{x}_{1}\right|+\left|\delta^{*}\right|\right)^{3}\right),
$$

where

$$
\begin{aligned}
h_{1}= & \frac{\lambda_{2}-a_{11}}{\lambda_{2}+1}\left[a_{12} a_{14}-a_{13}\left(1+a_{11}\right)\right]-\frac{\left(1+a_{11}\right)\left[a_{24}\left(1+a_{11}\right)-a_{12} a_{23}\right]}{\lambda_{2}+1}, \\
h_{2}= & \frac{\lambda_{2}-a_{11}}{a_{12}\left(\lambda_{2}+1\right)}\left(b_{1} a_{12}-b_{2}\left(1+a_{11}\right)\right)-\frac{a_{12} c_{2}-c_{1}\left(1+a_{11}\right)}{\lambda_{2}+1}, \\
h_{3}= & \frac{\lambda_{2}-a_{11}}{a_{12}\left(\lambda_{2}+1\right)}\left[a_{13} a_{12} a_{2}\left(\lambda_{2}-1-2 a_{11}\right)+2 a_{14} a_{12}^{2} a_{2}+b_{1} a_{1} a_{12}+a_{1} b_{2}\left(\lambda_{2}-a_{11}\right)\right. \\
& \left.-b_{3} a_{12}\left(1+a_{11}\right)+b_{4} a_{12}^{2}\right]-\frac{1}{\lambda_{2}+1}\left[( 1 + a _ { 1 1 } ) \left(a_{12} c_{3}+2 a_{2} a_{24}\left(\lambda_{2}-a_{11}\right)+a_{2} a_{12} a_{23}\right.\right. \\
& \left.\left.+c_{4}\left(1+a_{11}\right)\right)+\left(a_{1} c_{1}+a_{2} a_{12} a_{23}\right)\left(\lambda_{2}-a_{11}\right)+a_{1} c_{2} a_{12}\right], \\
h_{4}= & \frac{\lambda_{2}-a_{11}}{a_{12}\left(\lambda_{2}+1\right)}\left[a_{2} b_{2}\left(\lambda_{2}-a_{11}\right)+a_{12} a_{2} b_{1}-c_{1} a_{2} a_{12}\right]-\frac{a_{2} a_{12} c_{2}}{\lambda_{2}+1}, \\
h_{5}= & \frac{\left(\lambda_{2}-a_{11}\right)}{\lambda_{2}+1}\left[2 a_{12} a_{14}+a_{1} a_{13}\left(\lambda_{2}-a_{11}\right)-\left(a_{1} a_{13}+a_{12} a_{15}\right)\left(1+a_{11}\right)-a_{1} a_{12} a_{23}\right] \\
& -\frac{\left(1+a_{11}\right)}{\lambda_{2}+1}\left[a_{12} a_{25}\left(1+a_{11}\right)-a_{12} a_{23}-2 a_{1} a_{24}\left(\lambda_{2}-a_{11}\right)\right] .
\end{aligned}
$$

In order for map (3.4) to undergo a flip bifurcation, we require that two discriminatory quantities $\widetilde{\alpha}_{1}$ and $\widetilde{\alpha}_{2}$ are not zero, where

$$
\widetilde{\alpha}_{1}=\left.\left(G_{\widetilde{x}_{1} \delta^{*}}+\frac{1}{2} G_{\delta^{*}} G_{\widetilde{x}_{1} \widetilde{x}_{1}}\right)\right|_{(0,0)}=h_{2}
$$

and

$$
\widetilde{\alpha}_{2}=\left.\left(\frac{1}{6} G_{\widetilde{x}_{1} \widetilde{x}_{1} \widetilde{x}_{1}}+\left(\frac{1}{2} G_{\widetilde{x}_{1} \widetilde{x}_{1}}\right)^{2}\right)\right|_{(0,0)}=h_{5}+h_{1}^{2} .
$$

From a simple calculation, we obtain

$$
\widetilde{\alpha}_{1}=-\frac{2 \sqrt{\triangle_{1}}}{\left(\lambda_{2}+1\right)} \neq 0
$$

Thus, according to the above analysis and the theorem in $[16,17]$, we obtain the following result. 


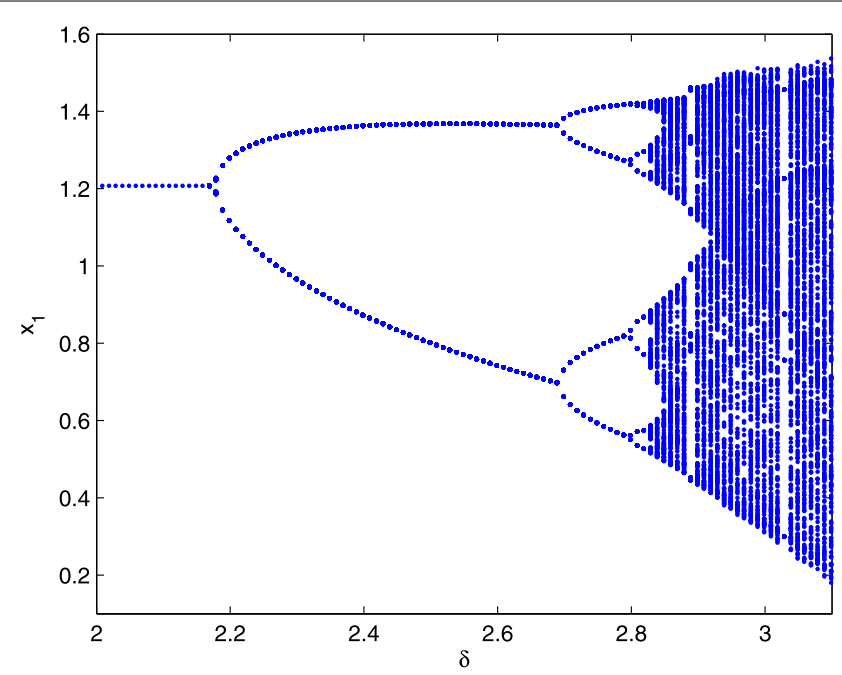

(a)

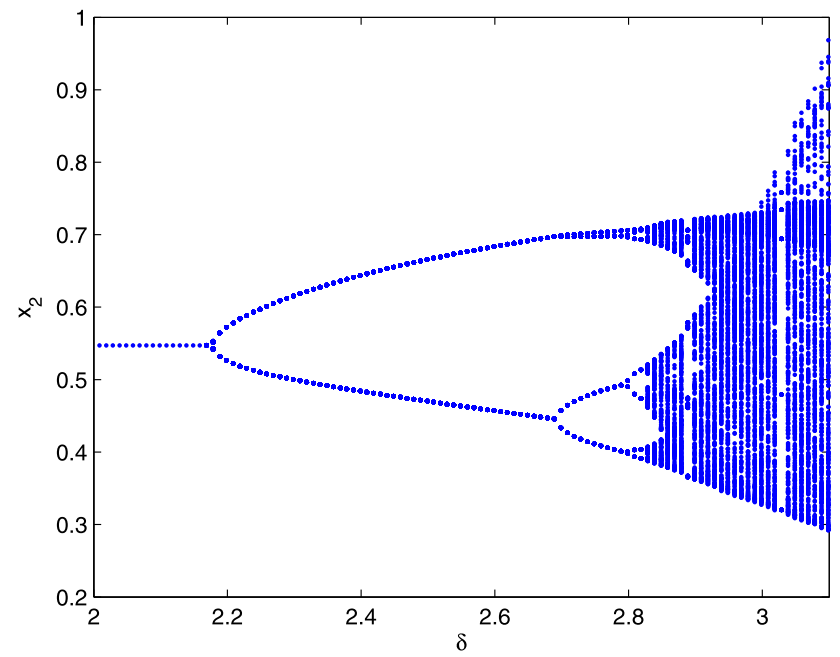

(b)

Figure 1 (a) Bifurcation diagram of $x_{1}$ for system (1.3) with $\delta$ ranging over [2, 3.1]. (b) Bifurcation diagram of $x_{2}$ for system (1.3) with $\delta$ ranging over $[2,3.1]$. (c) Maximum Lyapunov exponents corresponding for system (1.3) with $\delta$ ranging over [2, 3.1]

Theorem 3.1 If $\widetilde{\alpha}_{2} \neq 0$, then the map (3.1) undergoes a Flip bifurcation at the fixed point $E\left(x_{1}^{*}, x_{2}^{*}\right)$ when the parameter $\delta^{*}$ varies in the small neighborhood of the origin. Moreover, if $\widetilde{\alpha}_{2}>0$ (resp., $\widetilde{\alpha}_{2}<0$ ), then the period-2 points that bifurcate from $E\left(x_{1}^{*}, x_{2}^{*}\right)$ are stable (resp., unstable).

\section{Numerical simulations}

In this section, we present the bifurcation diagrams, phase portraits, and maximum Lyapunov exponents for the system (1.3) to confirm the above theoretical analysis and show the new interesting complex dynamical behaviors by using numerical simulations. The bifurcation parameters are considered for the following parameters.

Choosing $\alpha_{1}=0.5, \alpha_{2}=0.4, \beta_{12}=0.3, \beta_{21}=0.25, K_{1}=0.9, K_{2}=0.6, \gamma_{1}=0.2, \gamma_{2}=0.12$, initial value $\left(x_{1}(0), x_{2}(0)\right)=(0.5,0.3)$ and varying $\delta$ in the range $2 \leq \delta \leq 3.1$. 


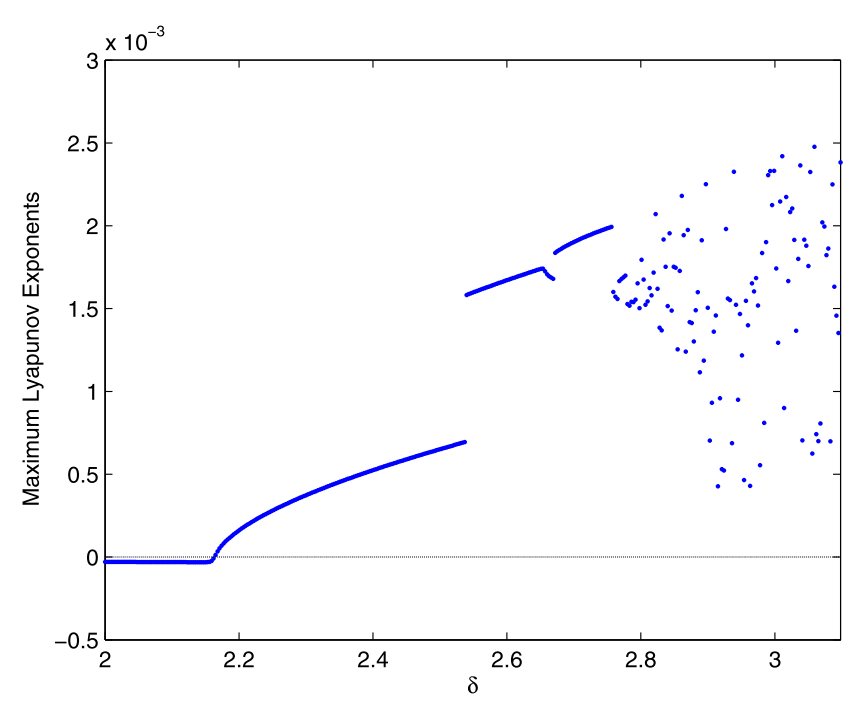

(c)

Figure 1 Continued.
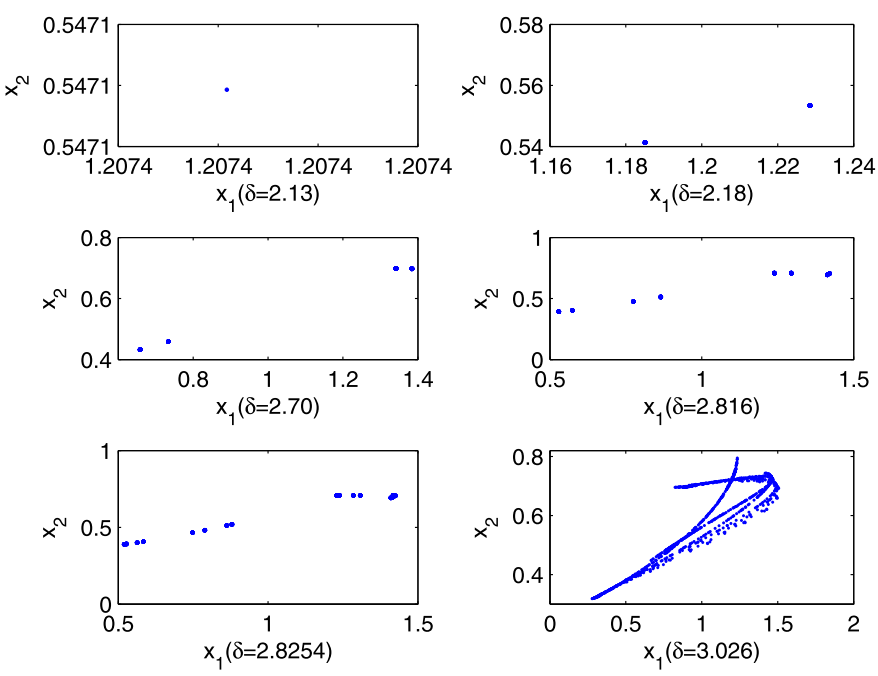

Figure 2 The phase portraits corresponding to Figures $1(a)$ and (b).

We see that the system (1.3) has only one positive fixed point, $(1.2074,0.5471)$. After calculation, by Theorem (3.1), the flip bifurcation emerges from the fixed point $(1.2074,0.5471)$ at $\delta=2.1786$ with $\alpha_{1}=-2.1462$ and $\alpha_{2}=5.2215$.

From Figures 1(a) and (b), we see that the fixed point $\left(x_{1}^{*}, x_{2}^{*}\right)$ is stable for $\delta<2.1786$, and that it loses its stability at the flip bifurcation parameter value $\delta=2.1786$. We also observe that there is a cascade of period doubling. The maximum Lyapunov exponents corresponding to Figures 1(a) and (b) are computed in Figure 1(c).

The phase portraits which are associated with Figures 1(a) and (b) are exposed in Figure 2. For $\delta \in(2.18,2.8254)$, there are orbits of period $2,4,8$, and 16 . When $\delta=3.026$, we 
can see the chaotic sets. The maximum Lyapunov exponent corresponding to $\delta=3.026$ is larger than 0 , which confirms the existence of chaotic sets.

\section{Discussion}

We can know that the dynamics of the system (1.2) is trivial with the condition (2.1). In fact, Samanta [19] has shown that the unique positive equilibrium of the system (1.2) is globally asymptotically stable with the condition (2.1). However, the discrete-time system (1.3) has complex dynamics. In this paper, we show that the unique positive fixed point of the system (1.3) can undergo a flip bifurcation with the condition (2.1). Moreover, numerical simulations display interesting dynamical behaviors for the system (1.3), including period-doubling orbits and chaotic sets.

\section{Competing interests}

The authors declare that they have no competing interests.

\section{Authors' contributions}

All authors read and approved the final manuscript.

\section{Author details}

${ }^{1}$ Department of Mathematics, Anqing Normal University, Anqing, Anhui 246133, China. ${ }^{2}$ Department of Mathematics, and Research Center for Complex Systems and Network Sciences, Southeast University, Nanjing, Jiangsu 210096, China.

\section{Acknowledgements}

This work is jointly supported by the Programs of Educational Commission of Anhui Province of China under Grant nos. KJ2011A197 and KJ2013Z186.

Received: 16 September 2013 Accepted: 7 February 2014 Published: 19 Feb 2014

\section{References}

1. Nozawa, K: The effect of peridinium toxin on the algae. Bull. Misaki Mar. Biol. Inst. Kyoto Univ. 12, 21 (1968)

2. Harris, DO: A model system for the study of algal growth inhibitors. Arch. Protistenkd. 113, 230 (1971)

3. Huntsman, SA, Barber, RT: Modification of phytoplankton growth by excreted compounds in low-density populations. J. Phycol. 11, 10 (1975)

4. Mukhopadhyay, A, Chattopadhyay, J, Tapaswi, PK: A delay differential model of plankton allelopathy. Math. Biosci. 149, 167-189 (1998)

5. Wolfe, JM, Rice, EL: BRCA1 protein products: functional motifs. Nat. Genet. 13, 266-267 (1996)

6. Rice, EL: Allelopathy. Academic Press, New York (1984)

7. Maynard, J: Models in Ecology. Cambridge University Press, Cambridge (1974)

8. Chattopadhyay, J: Effect of toxic substances on a two-species competitive system. Ecol. Model. 84, $287-289$ (1996)

9. Chen, FD: Permanence in a discrete Lotka-Volterra competition model with deviating arguments. Nonlinear Anal., Real World Appl. 9, 2150-2155 (2008)

10. Chen, YM, Zhou, Z: Stable periodic solution of a discrete periodic Lotka-Volterra competition system. J. Math. Anal. Appl. 277, 358-366 (2003)

11. Chen, GY, Teng, ZD: On the stability in a discrete two-species competition system. J. Appl. Math. Comput. 38, 25-36 (2012)

12. Kong, XZ, Chen, LP, Yang, WS: Note on the persistent property of a discrete Lotka-Volterra competition system with delays and feedback controls. Adv. Differ. Equ. 2010, 249364 (2010)

13. Huo, HF, Li, WT: Permanence and global stability for nonautonomous discrete model of plankton allelopathy. Appl. Math. Lett. 17, 1007-1013 (2004)

14. Qin, WJ, Liu, ZJ: Asymptotic behaviors of a delay difference system of plankton allelopathy. J. Math. Chem. 48, 653-675 (2010)

15. Liu, ZJ, Chen, LS: Positive periodic solution of a general discrete non-autonomous difference system of plankton allelopathy with delays. J. Comput. Appl. Math. 197, 446-456 (2006)

16. Gukenheimer, J, Holmes, P: Nonlinear Oscillations, Dynamical Systems, and Bifurcation of Vectro Fields. Springer, New York (1983)

17. Wiggins, S: Introduction to Applied Nonlinear Dynamical System and Chaos. Springer, New York (2003)

18. Carr, J: Application of Center Manifold Theory. Springer, New York (1981)

19. Samanta, GP: A two-species competitive system under the influence of toxic substances. Appl. Math. Comput. 216, 291-299 (2010)

10.1186/1687-1847-2014-70

Cite this article as: Wu and Zhang: Bifurcation analysis of a two-species competitive discrete model of plankton

allelopathy. Advances in Difference Equations 2014, 2014:70 